\title{
Addressing inequities in access to quality health care for indigenous people
}

\author{
David Peiris MBBS MIPH, Alex Brown BMed MPH, Alan Cass MBBS PhD
}

$\infty$ See related research paper by Gao and colleagues, page 1007

$\mathrm{M}$ any issues influence access to quality health care for indigenous people. In this issue of $C M A J$, Gao and colleagues ${ }^{1}$ describe inequities in access to health care and service utilization among Canadian Aboriginal people with chronic kidney disease. Similar findings have been reported in Australia, ${ }^{2}$ New Zealand ${ }^{3}$ and the United States. ${ }^{4}$ Although well-conducted studies that quantify the extent of the disparity and trends in health care access are needed, addressing the underlying causes of this disparity is a priority - not merely because such disparities are unacceptable but because disparities in access contribute to major and avoidable ill health.

One key contextual barrier to accessing health care that has been described in the literature from Australia, Canada, New Zealand and the US is the continuing impact of colonization. ${ }^{5}$ The Canadian Royal Commission on Aboriginal Peoples and the Australian Royal Commission on Aboriginal Deaths in Custody comprehensively documented the contemporary effects of past discriminatory policies on indigenous people. ${ }^{6,7}$ Although few empirical studies have examined the health effects of discriminatory policies, a well-conducted cohort study in Australia reported that the forced removal of Aboriginal children from their families affected health for generations. ${ }^{8}$ By engendering distrust in government agencies, policies such as these contribute to high levels of stress among indigenous people. Psychosocial stress, a phenomenon common to many vulnerable populations, is an important barrier to accessing health care and has been consistently associated with adverse health outcomes for indigenous people. ${ }^{9}$

Health care systems and health care services are not immune from this historical policy context. Studies, predominantly with qualitative designs, have shown that indigenous people are sensitive to power imbalances in their interactions with health care services. This is intimately linked with the dominance of the biomedical paradigm and the view that noncompliant behaviours by indigenous people are the cause of poor health outcomes. By contrast, when care providers promote a nonbiomedical approach to health care interactions, through trust, reciprocity and shared decision-making, they can empower recipients and more effectively deliver interventions to reduce the gap in health outcomes. Much work focuses on miscommunication as an access barrier. ${ }^{10}$ Relevant factors include communication dynamics and sharing of health information, language and literacy. In health care for indigenous people, the power dynamic directly affects communication.

A related, complex area is the attribution of cultural factors as both barriers to and facilitators of health care. There is a clear need to abandon stereotypical concepts of indigenous cultures and the simplistic embrace of particular culture-based

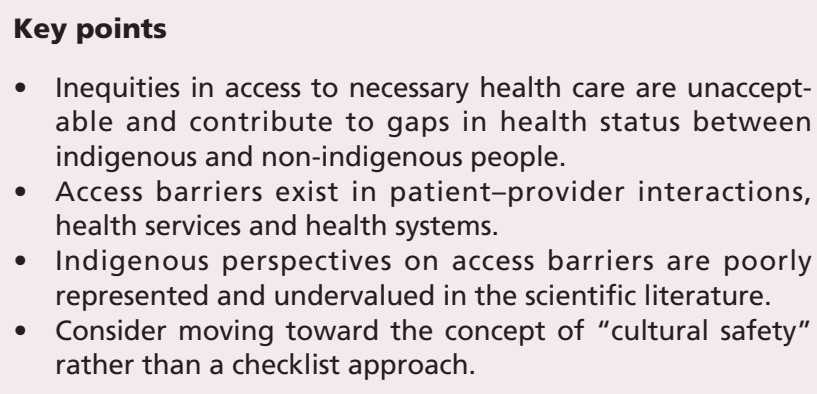

- Inequities in access to necessary health care are unacceptable and contribute to gaps in health status between indigenous and non-indigenous people.

- Access barriers exist in patient-provider interactions, health services and health systems.

- Indigenous perspectives on access barriers are poorly represented and undervalued in the scientific literature.

- Consider moving toward the concept of "cultural safety" rather than a checklist approach.

ingredients as means of transforming services into becoming "appropriate," "aware," "sensitive" or "competent" - terms that are often poorly defined. The more dynamic concept of "cultural safety," originally developed by Maori nurses, is quite different. ${ }^{11}$ Cultural safety shifts the role of culture away from a check-list approach based on a person's ethnic background and toward a critical examination of the power imbalances in health care encounters between indigenous patients and non-indigenous health care providers. When viewed in this way, culturally safe health care becomes a core principle for the reorientation of health services to better meet the needs of vulnerable groups, irrespective of their ethnic background.

We need to move beyond patient-provider interactions in developing a policy-informing agenda on access. Known facilitators of access are the establishment of community-governed health services, a robust indigenous managerial and clinical workforce and the ability to deliver models of care that embrace indigenous knowledge systems. ${ }^{12}$ The interpretive synthesis of the literature about the barriers to access for vulnerable groups by Dixon-Woods and colleagues has led to the development of the useful concepts of "navigation" and "permeability." ${ }^{13}$ Navigation requires an awareness of the available services and the mobilization of personal and health service resources to provide access, such as transport, minimal out-of-pocket cost and flexible hours. Permeable services require little negotiation for entry and a minimal level of understanding of how the system works. These services may include having welcoming physical spaces, open-door policies and reception staff who are known to the community.

Measures of health system performance are increasingly used to improve access and quality of care. The US Indian Health Service has invested substantially in information tech-

From the George Institute for International Health (Peiris, Cass), Sydney; the Centre for Indigenous Vascular and Diabetes Research (Brown), Baker IDI Heart and Diabetes Institute, Alice Springs; The Poche Centre for Indigenous Health (Cass), University of Sydney, Sydney, Australia 
nology to support macrosystem monitoring of quality measures. $^{14}$ The Australian government is developing quality indicator programs for Aboriginal health services. Although these approaches are valuable, caution is needed in their application. In a review of indigenous health performance measurement systems, Smylie and colleagues argue that the development of these macrosystem measures, which are usually based on physical and disease variables, often come at the expense of developing locally specific health indicators for indigenous populations. ${ }^{15}$ The article by Gao and colleagues exemplifies the problem discussed by Anderson and colleagues ${ }^{5}$ in the classification of ethnicity, particularly for people of mixed descent. The classification of non-status First Nations and Metis people as non-Aboriginal is epidemiologically expedient, but it raises concerns about equity. Support for local measurement systems responsive to indigenous people and to their health service priorities can complement conventional performance measures and ensure valid interpretation of those measurements.

Our list of barriers and facilitators is not exhaustive. A key consideration is the need for greater clarity in how we conceptualize barriers, their defining characteristics and their causes. Closely related is the need to move beyond explanatory models, which focus on deficiencies in individuals and services, and toward a better understanding of existing facilitators to improved access and quality. The research on which to base policy is a labyrinthine mix of qualitative studies, audits and surveys, program evaluations, advocacy, quality improvement projects, nonsystematic topic reviews and opinion pieces. Opinion pieces are often key sources of indigenous perspectives but are poorly represented and undervalued in the scientific literature. Much of this disparate literature is locally specific, small in scale and is often not amenable to the reductionism of conventional systematic reviews. This makes national and international extrapolation difficult and poses dilemmas for the development of evidence-based policy.

In Canada, Australia, New Zealand and the US, inequities in access to health care and outcomes for indigenous people have been well-characterized. To close these gaps, we need to document disparities and understand their causes more precisely and make collaborative changes. Such changes are often resisted by institutional, political, structural, social and cultural forces. As indicated by an Aboriginal man with endstage kidney disease interviewed for the Improving Access to Kidney Transplants study ${ }^{16}$ when discussing communication with his kidney specialists: "You don't go knocking on their door, [that's the] 'danger one.' The door is locked. They sit behind closed doors." If we are able to open these doors, our health care systems may perform better for the most vulnerable.

Competing interests: None declared.

Contributors: Each of the authors contributed to the content of the article, revised it critically and approved the final version for publication.

Acknowledgements: We thank Dr. Peter Arnold for his help in editing this article.

David Peiris is supported by a scholarship from the New South Wales Clinical Excellence Commission. Alan Cass is the recipient of a National Health and Medical Research Council Senior Research Fellowship.

\section{REFERENCES}

1. Gao S, Manns BJ, Culleton BF, et al. Access to health care among status Aboriginal people with chronic kidney disease. CMAJ 2008;179:1007-12.

2. Deeble J, Agar JS, Goss J. Expenditures on health for Aboriginal and Torres Strait Islander peoples 2004-05. Canberra (Australia): Australian Institute of Health and Welfare; 2008. Available: www.aihw.gov.au/publications/hwe/eohfatsip04-05 leohfatsip04-05.pdf (accessed 2008 Oct 1).

3. Tukuitonga $\mathrm{CF}$, Bindman $\mathrm{AB}$. Ethnic and gender differences in the use of coronary artery revascularisation procedures in New Zealand. N Z Med J 2002;115:179-82.

4. Zuckerman S, Haley J, Roubideaux Y, et al. Health service access, use, and insurance coverage among American Indians/Alaska Natives and whites: What role does the Indian Health Service play? Am J Public Health 2004;94:53-9.

5. Anderson I, Crengle S, Kamaka ML, et al. Indigenous health in Australia, New Zealand, and the Pacific. Lancet 2006;367:1775-85.

6. Johnston E. Royal Commission on Aboriginal deaths in custody: national report volume 2. Canberra (Autralia): Australian Government Publishing Service 1991. Available: www.austlii.edu.au/au/special/rsjproject/rsjlibrary/rciadic/national/vol2/ (accessed 2008 Oct 1 )

7. Report of the Royal Commission on Aboriginal People. Volume 3: Gathering strength. Ottawa (ON): Indian and Northern Affairs Canada; 1996. Available: www.ainc-inac.gc.ca/ch/rcap/sg/si1_e.html\#Volume\%203 (accessed 2008 Oct 1).

8. Zubrick SR, Silburn SR, Lawrence DM, et al. The Western Australian Aboriginal Child Health survey: the social and emotional wellbeing of Aboriginal children and young people. Perth (Australia): Curtin University of Technology and Telethon Institute for Child Health Research; 2005. Available: www.ichr.uwa .edu.au/waachs/publications/volume_two (accessed 2008 Oct 1 ).

9. Paradies Y. A review of psychosocial stress and chronic disease for 4th world indigenous peoples and African Americans. Ethn Dis 2006;16:295-308.

10. Cass A, Lowell A, Christie M, et al. Sharing the true stories: improving communication between Aboriginal patients and healthcare workers. Med J Aust 2002;176:466-70.

11. Papps E, Ramsden I. Cultural safety in nursing: the New Zealand experience. Int J Qual Health Care 1996;8:491-7.

12. Crampton P, Davis P, Lay-Yee R, et al. Does community-governed nonprofit primary care improve access to services? Cross-sectional survey of practice characteristics. Int J Health Serv 2005;35:465-78.

13. Dixon-Woods M, Cavers D, Agarwal S, et al. Conducting a critical interpretative synthesis of the literature on access to health care by vulnerable groups. BMC Med Res Methodol 2006;6:35.

14. Sequist TD, Cullen T, Ayanian JZ. Information technology as a tool to improve the quality of American Indian health care. Am J Public Health 2005;95:2173-9.

15. Smylie J, Anderson I, Ratima M, et al. Indigenous health performance measurement systems in Canada, Australia, and New Zealand. Lancet 2006;367:2029-31.

16. Devitt J, Cass A, Cunningham J, et al. Study protocol - Improving Access to Kidney Transplants (IMPAKT): A detailed account of a qualitative study investigating barriers to transplant for Australian Indigenous people with end-stage kidney disease. BMC Health Serv Res 2008;8:31.

Correspondence to: Dr. Alan Cass, The George Institute for International Health, PO Box M201, Missenden Rd., Sydney NSW 2050, Australia; fax 6129993 4502; acass@george.org.au 